\title{
ANALYSIS OF THE COMPRESSION RATIO AND QUALITY IN AERIAL IMAGES
}

\author{
D. Mateika, R. Martavičius
}

Department of Electronic Systems, Vilnius Gediminas Technical University, Naugarduko 41, LT-03227 Vilnius, Lithuania.E-mail: romanas.martavicius@el.vgtu.lt

Received 29 June 2006, accepted 10 Oct 2007
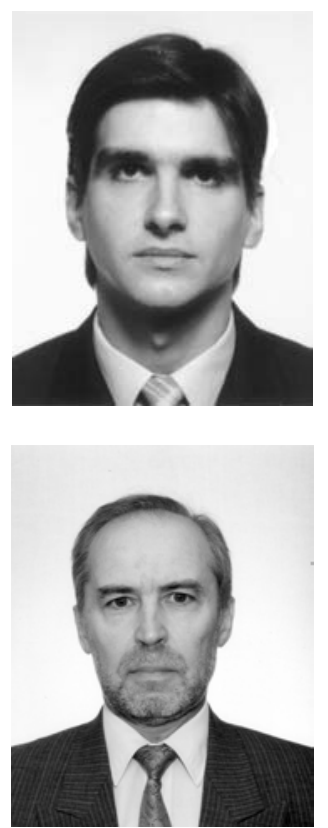

Darius MATEIKA, PhD student

Date and place of birth: Molètai, Lithuania, 1980.

Education: Faculty of Electronics, Vilnius Gediminas Technical University, M.Sc. (2004).

Fields of research: information management, shared document usage and creation systems.

Present position: PhD student in the Faculty of Electronics; Vilnius Gediminas Technical University.

\section{Romanas MARTAVIČIUS, Prof Dr Habil}

Date and place of birth: Kaunas, Lithuania, 1945.

Education: Vilnius branch of Kaunas Polytechnical Institute (later VTU); Radio Engineering Diploma (1968),

Doctor Sc. Eng. Degree (1977), 1984. Doctor Habilitus degree in Eng. (1997).

Fields of research: computerized electronic systems and IT methods, analysis and recognition of medical and other images.

Present position: head of Department of Electronics Systems, professor at Vilnius Gediminas Technical

University, and member of the Institute of Electrical and Electronics Engineers (IEEE).

Publications: monographs: "Super-wide band tracts of the traveling-wave cathode-ray tubes" (1993),

"Electrodynamic plain retard systems for wide band electronic devices" (1996), textbook: "Analog Electronics"

(2003) and more than 100 scientific papers.

Abstract. In modern photomap systems, images are stored in centralized storage. Choosing a proper compression format for the storage of an aerial image is an important problem. This paper analyses aerial image compression in popular compression formats. For the comparison of compression formats, an image quality evaluation algorithm based on the calculation of the mean exponent error value is proposed. An image quality evaluation experiment is presented. The distribution of errors in aerial images and explanation of the causes for worse than usual compression effect are analysed. An integrated solution for the aerial image compression problem is proposed and the compression format most suitable for aerial images is specified.

Keywords: compression, quality, algorithm, digital images, photomap.

\section{Introduction}

It is important to document images in aviation. Later on, documents must be stored in archives. When images are taken at a high altitude, they must have a large amount of pixels to store precise information [2, 5]. Highresolution images require big memory capacities. Compressing images can reduce capacity. This paper analyses the most popular image compression methods: JPEG, JPEG2000, PNG, GIF, LZW, and Run-length encoding (RLE). According to quality, image compression algorithms can be divided into two groups:

- Algorithms that compress images without data loss.
- Algorithms that compress images with data loss.

PNG, LZW and RLE belong to the first group $[4,6,7]$. Such images can be restored to their original state at any time. The GIF format also saves images without data loss under one condition: images must be monochromatic with 8-bit intensity depth. JPG always loses data when compressing images [8]; JPG 2000 can perform in both ways. Loss image compression gives a higher ratio.

In modern aerial information management systems image, compression storages meet dual and contradictory requirements. The content of the aerial images must be saved with minimal information loss and the highest compression ratio because of big size. 
This paper describes the search for a compromise solution to identify the image compression method giving the highest compression ratio and the smallest image information loss. For this purpose, comparing compression methods using mean exponential error criteria is proposed. During analysis, selected image compression formats are analysed experimentally. The most appropriate compression solution for the aerial images is identified and proposed.

\section{Algorithm that compares image compression quality}

There are two different image compression methods: image compression with quality loss and without quality loss $[1,3]$.

Images compressed using the second method can be restored to their previous state without distortion at any time. The first group of image compression methods compresses with a better ratio, but there are always differences after an image is restored from its compressed state. These differences can be major or minor. In many image usage cases, small differences are acceptable. When it is required to say whether differences are acceptable or not, distortion should be measured.

One of the ways to measure differences between an original and a compressed image is to calculate mean exponential error. This method was chosen because it accumulates exponential error from every image pixel. This means that even the smallest differences will be evaluated. Mean exponential error is described by the following equation:

$$
D=\frac{1}{N} \sum_{i=1}^{N} e^{\mid\left(A_{i}-A_{g i}\right)} \mid
$$

where: $D$ - mean exponential error, $N$ - number of pixels in the image, $A$ - intensity of pixel i from the original image, $A_{g i}-$ intensity of pixel $\mathrm{i}$ from the compressed image.

Images are originally rectangular. For real images, this expression must be modified. Rectangular images have two dimensions: $\mathrm{X}$ and $\mathrm{Y}$. Adding one additional dimension for the (1.1) modifies it in this manner:

$$
D=\frac{1}{X} \frac{1}{Y} \sum_{i=1}^{X} \sum_{j=1}^{Y} e^{\mid\left(A_{i j}-A_{g i j}\right)} \mid
$$

where: $D$ - mean exponential error, $X$ - image width, $Y-$ image height, $A_{i j}$ - intensity of pixel $i j$ from the original image, $A_{g i j}$ - intensity of pixel $i j$ from the compressed image.

Expression (2) can already be used for the calculation of mean exponential error in gray images. But pixels of colour images require more than one value for the description of the exact colour. For this purpose, in colour images, colour bands are used. For example, in colour coding system, RGB, there is three colour bands: red, green and blue. The exact colour of the image pixel is described by the combination of intensities from each colour band. Gray images have only one band, and RGB has three. There is also colour-coding systems with four or more bands. Expression (3) adapted for colour images looks like this:

$$
D=\frac{1}{X} \frac{1}{Y} \frac{1}{C} \sum_{i=1}^{X} \sum_{j=1}^{Y} \sum_{k=1}^{C} e^{\mid\left(A_{i j k}-A_{g i j}\right)} \mid
$$

where: $D$ - mean exponential error, $X$ - image width, $Y-$ image height $C$ - number of colour bands, $A_{i j k}$ - intensity of pixel $i j$ in colour band $k$ from the original image, $A_{g i j k}-$ intensity of pixel $i j$ in colour band $k$ from the compressed image.

\section{Implementation of the image comparison algorithm}

At the beginning, the implementation must read the parameters of the image. This means that it must determine image width, height, and number of bands. Image width and height sets the size of the twodimensional image intensity data array each colour band has its own array. When comparing two images of a gray type, there will be two arrays. In the case of RGB colour images, there will be six arrays.

When the structure of the image is identified, the implementation must read the image data. The result of this action is two sets of pixel intensity arrays of the same structure.

Block (2) in figure 1 illustrates the calculation of the difference in pixel intensity. Corresponding pixel intensity values in the original and compressed images are subtracted. The result of this action is a third set of pixel intensity arrays.

The differences that are detected are revealed in block (3). The exponent of base $e$ is calculated for each pixel. This operation highlights small differences in values that are also important but hard to detect. The image formed from the results of this calculation is called an error map. The map is shown in figures 2 and 3. In figure 2 differences are hardly visible, whereas in Figure 3 the differences are clear. Without such an operation, small error values have a similar low intensity and the human eye simply cannot recognize them.

The next algorithm block (4) calculates the final product - mean exponent error. All image pixel intensities from all colour bands are summed up and the number of pixel in the image divides the result. The final result of such calculations is one number that represents image quality. A value of 1.0 means that the two images are identical, and higher values mean bigger differences between the images. 


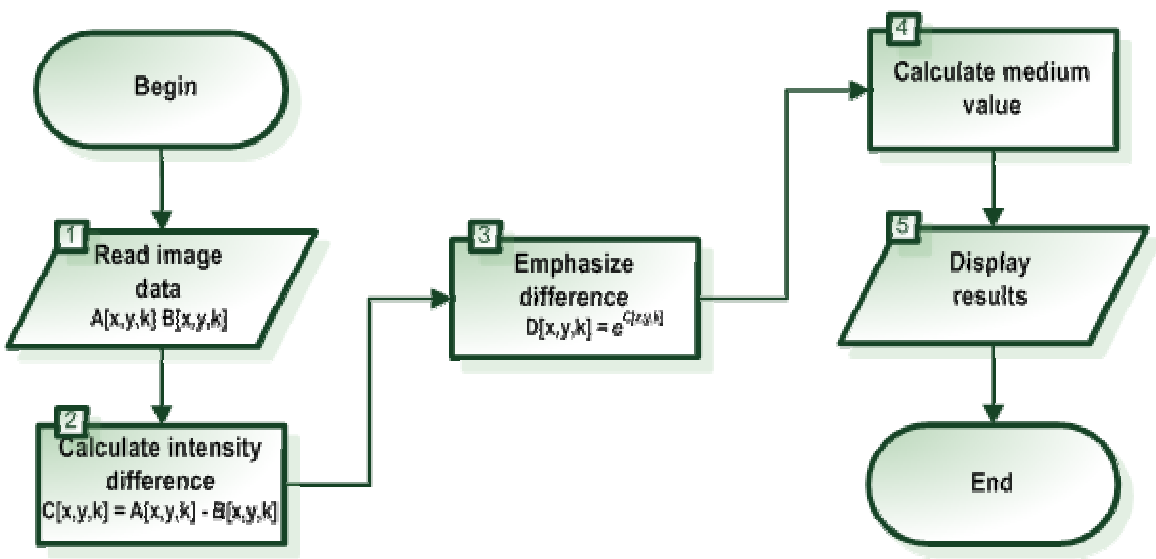

Fig 1. Algorithm that compares image compression quality

\section{Background of analysis of image compression quality}

Several digital image-coding formats were analysed using software based on the algorithm described in the previous section. The goal of the analysis was to determine which compression algorithm saves images with higher quality at higher compression ratios. These image-coding formats were used in the analysis:

- JPG

- JPG 2000 GIF

- PNG

- TGA (with RLE compression)

- TIF (with LZW compression)

Two image sets were selected for the experiment. One set was a monochromatic aerial photo. These photos were taken with a digital camera in an uncompressed format from the airplane. The images contain urbanized areas, forests, mountains, and bodies of water. Before analysis these images were converted to 8-bit intensity depth, which is usual in photomap information systems.

Colour images selected for analysis are also uncompressed 24-bit BMP images. Images were acquired with a digital photo camera by taking pictures of the surroundings. Both image sets used had the same total size.

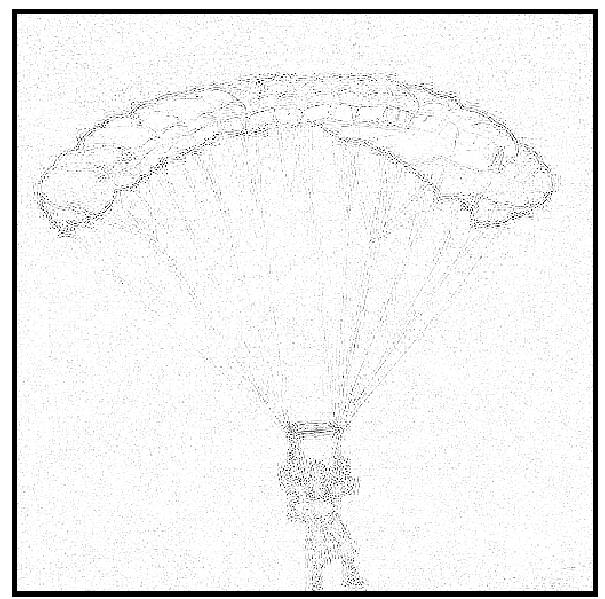

Fig 2. Image intensity difference map

\section{Results of analysis of image compression quality}

Before analysis, sample images were converted to several different image storage formats. That formats that were selected use different image compression algorithms. The most interesting results of the analysis were:

- Image quality.

- Image file size.

In reference to quality, image compression algorithms can be divided into two groups:

- Algorithms that compress images without data loss.

- Algorithms that compress images with data loss.

PNG, TIF/LZW and TGA/RLE belong to the first group. GIF format also saves images without data loss under one condition: images must be monochromatic with 8-bit intensity depth. The results of calculations are presented in tables 1 and 2. According to this data, aerial images were compressed with lower ratios

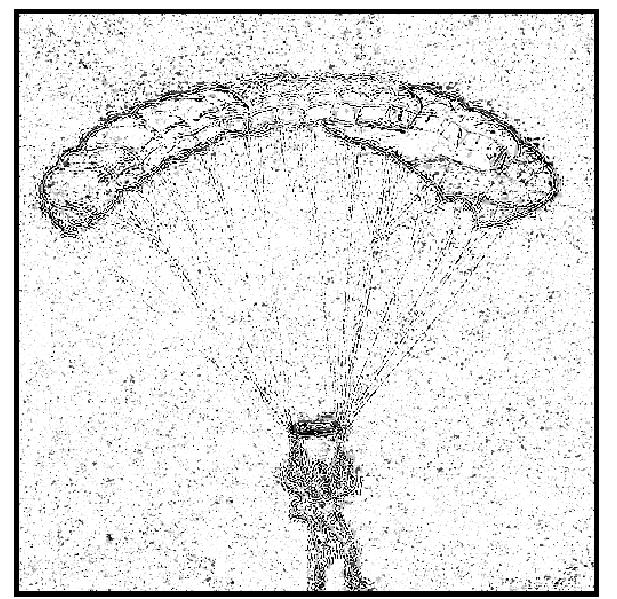

Fig 3. Exponential image intensity difference map 
Table 1. Results of analysis of monochrome image compression

\begin{tabular}{|c|r|r|r|}
\hline Format & Size KB & Size $\%$ & Error \\
\hline BMP & 3752 & 100 & 1.0 \\
\hline GIF & 3961 & 106 & 1.0 \\
\hline PNG & 3313 & 88 & 1.0 \\
\hline JP2 (bp) & 2204 & 59 & 1.0 \\
\hline TIFF/LZW & 3989 & 106 & 1.0 \\
\hline TGA/RLE & 3922 & 105 & 1.0 \\
\hline JP2 (100) & 2204 & 59 & 1.0 \\
\hline JPG (100) & 2382 & 63 & 1.1 \\
\hline JP2 (66) & 157 & 4 & 57.5 \\
\hline JPG (66) & 530 & 14 & 32.2 \\
\hline JP2 (33) & 56 & 2 & 73.5 \\
\hline JPG (33) & 326 & 9 & 48.4 \\
\hline
\end{tabular}

in all cases. The best compression ratio without data loss is given by JPG 2000. Images stored in this data format need only approximately $60 \%$ of the space required by uncompressed data. The second best result was returned by PNG - approximately $90 \%$. This means that an aerial image compressed with such a method saves only $10 \%$ of the space that would be used by an uncompressed image. Compressing generic colour images with the same method (PNG) gives a ratio equal to JPEG 2000 with aerial images. GIF, TIFF/LZW and TGA/RLE do not give any positive effect at all. Compressed images require even more space than those in an uncompressed state. Such compression algorithms in this case are not effective and additional space is taken by service data.

In the case of generic colour images, GIF already yields errors. The ratio of JPG 2000 errors is about 10 percent higher than it was for aerial images. TIFF/LZW and TGA/RLE methods are also useless with generic colour images, but images compressed in such a manner at least do not require more space than was needed in the original state.

As can be seen from the test results, loss less aerial image compression does not give any reasonable result. Photomap systems do not require as much accuracy as, for example, medical images. In medicine distorted images are not allowed. The cost of an incorrect medical decision made after analysing distorted a medical image is too high. An incorrectly interpreted aerial image also can have big cost in terms of money, but there is no risk to human life. In this case, lossy compression with a controlled level of distortion is acceptable. Compressing images with loss compression formats gives much better ratios. JPEG format is always lossy. Storing aerial images in JPEG at its best quality already yields small errors, and the results are worse than in the case of loss less JPG 2000. Setting lower quality in the JPEG format gives much higher compression ratios. For example, saving images in JPEG at a quality of $66 \%$ compresses image size approximately five times, at $33 \%$ about 10 times. Results are very similar when comparing quality losses of JPG and JPG 2000. Results of lossy colour image compression are a bit different. Exponential error at the
Table 2. Results of analysis of colour image compression

\begin{tabular}{|c|r|r|r|}
\hline Format & Size KB & \multicolumn{1}{|c|}{ Size $\%$} & \multicolumn{1}{c|}{ Error } \\
\hline BMP & 3840 & 100 & 1.0 \\
\hline GIF & 749 & 19 & 46.5 \\
\hline PNG & 2216 & 57 & 1.0 \\
\hline JP2 (bp) & 1806 & 47 & 1.0 \\
\hline TIFF/LZW & 3873 & 99 & 1.0 \\
\hline TGA/RLE & 3756 & 96 & 1.0 \\
\hline JP2 (100) & 1806 & 47 & 1.0 \\
\hline JPG (100) & 1047 & 27 & 10.7 \\
\hline JP2 (66) & 163 & 4 & 38.2 \\
\hline JPG (66) & 199 & 5 & 42.0 \\
\hline JP2 (33) & 60 & 2 & 50.8 \\
\hline JPG (33) & 122 & 3 & 51.5 \\
\hline
\end{tabular}

same ratios is lower. According to the results of our analysis, images compressed with JPEG at the same percentage of quality give the same error level regardless of size. The situation is the opposite for JPEG 2000; the error level changes while the compression ratio are the same.

The results of the analysis clearly show that aerial images are compressed with a lower compression ratio and a higher error level.

The reason for the lower monochrome image compression ratio is visible in figures 3 and 4 , where exponential image compression error maps are presented. Generic images have slowly changing regions of intensity. As is visible in figure 3, the biggest compression error could be found in the regions where intensity is changing quickly. Such regions have a lot of small details. Image regions with low details are called smooth. Smooth regions could be out-of-focus objects in photography, big objects, or very close objects. In aerial photography in which pictures of the terrain are taken from an exact height, all objects are in focus and small because the distance is big. Because of this, all regions of aerial images have many details. A large number of details mean a high level of error in all regions. As visible in figure 4 , the error rate in all regions of the aerial images is very similar.

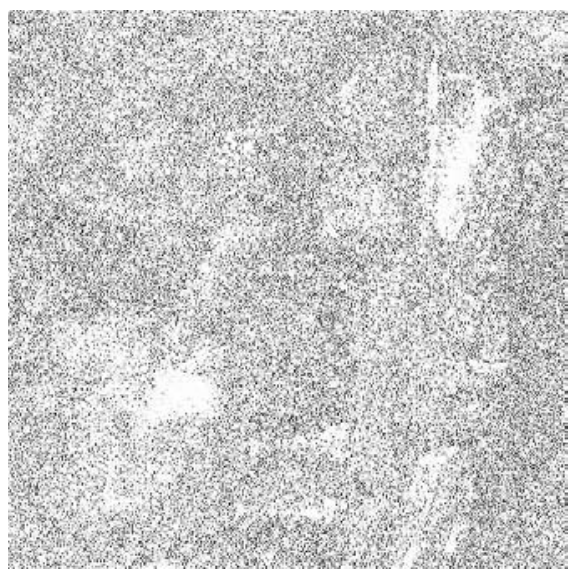

Fig 4. Exponential intensity difference map of the aerial image 
According to the results of the analysis, lossy image compression gives much higher ratios than loss fewer methods. As was mentioned before, controlled image distortion in photomap systems is acceptable. Lossy methods can be used in image transfers when channel bandwidth is low. Transferring an entire image compressed loss less with a ratio 2:1 may take too long, however. Some solutions in such a case could be:

1. Transferring a lossy compressed image as an image map. Ratios there can be 50:1 and higher and it is still possible to understand image content.

2. Selecting a region of interest.

3. Transferring a loss less image of a selected region.

4. Transferring the rest of an image as a background job.

Such workflow would require transferring excess data through the communication channel, but the decision based on the review of the transferred image could be made faster. Standard compression methods do not allow using such workflow without sending excess data, but it is possible to create a method that would allow the reuse of data already sent in step 1 for the rest of the transferring steps.

\section{Conclusion}

1. The method created to compare images detects even the smallest compression errors. This method gives two possible outcomes: a numerical value, which allows the similarity of images to be expressed in numbers and determine algorithm giving the best compression quality. Error map - which describes what image regions are compressed with biggest error and helps to analyse image compression specifics.

2. Image compression can be loss or loss less. The best results from the loss less compression group were shown by JPEG 2000. When compressing lossy image compression, results from JPEG and JPEG 2000 were actually the same.

3. Aerial images are specific. All objects in aerial images are in focus and are small because of the large distance. Because of that, such images are very sharp and have lots of details. Images with details should be compressed with a lower ratio and higher error level. All regions of the image have a similar level of detail and the level of compression error is also distributed throughout the image.

4. In aerial photomap systems, image distortion is acceptable. Loss less compression gives too low a ratio. According to the results of our analysis, the image storing loss less JPEG 2000 can achieve ratios of $\sim 1: 2$. Transferring images with such a ratio can take too long if the bandwidth of the channel is low. A solution to this problem could be transferring a lossy compressed image map. The ratio there can be approximately 1:50. The image map would allow users to selection of a region of interest, which should be transferred loss less first.

\section{References}

1. COSMAN, P., GRAY, R., OLSHEN, R. Evaluating quality of compressed medical images: SNR, subjective rating, and diagnostic accuracy. In Proceedings of the IEEE. 1994, vol. 82, p. 919-932.

2 DECK, J. Aerial imagery guidelines. In http://gis.csc.edu/Projects/aerial/guidelines.htm .

3. ESKICIOGLU, AM., FISHER, PS. Image quality measures and their performance. IEEE Transactions on Communications. 1995, vol. 43, p. 2959-2965.

4. FRÄNTI, P., NEVALAINEN O., KAUKORANTA T. Compression of digital images by block truncation coding: a survey. The Computer Journal. 1994, vol. 37, no. 4, p. 308-332.

5. GUNGOR, B., YASAYAN, A., DEMIREL, AS. et al. Aerial photograph archives information system. In Geo-Imagery Bridging Continents. XXth ISPRS Congress. Istanbul, 2004, p.12-23.

6. HANKERSON, D., HARRIS, GA., JOHNSON, PD. Jr. Introduction to information theory and data compression. Chapman \& Hall. CRC Press Company, 2003, p. 352.

7. Image file formats. In http://en.wikipedia.org/ wiki/image_formats.

8. WALLACE, GK. The JPEG still picture compression standard. Communications of the ACM. 1991, vol. 34, no. 4, p. 30-44. 